\title{
Lydia Flem : « L'imagination est ma seule maison ». Entretien autour des lettres (et) objets ${ }^{1}$
}

\author{
Karin Schwerdtner \\ Université Western Ontario \\ Canada
}

Écrivaine et psychanalyste, reconnue d'abord pour ses ouvrages biographiques tels que L'Homme Freud. Une biographie intellectuelle (1991) et Casanova ou l'exercice du bonheur (1995), puis pour la trilogie familiale que constituent ensemble Comment j'ai vidé la maison de mes parents (2004), Lettres d'amour en héritage (2006) et Comment je me suis séparée de ma fille et de mon quasi-fils (2009), Lydia Flem est devenue photographe en 2008, lorsqu'elle recevait des traitements induisant des difficultés d'attention et de concentration. Elle était alitée, incapable de lire et d'écrire plus de quelques phrases, comme elle le précise elle-même, dans cet entretien. Pour continuer à créer et essayer de raconter ce qu'elle ne pouvait ni taire ni exprimer avec des mots, elle a alors cherché à fixer en image des assemblages de choses à portée de main : des fragments de papier, des jouets et objets souvenirs, des aliments et des articles et ustensiles de la vie privée. « [M]êl[a]nt plantes et bijoux, papier et gingembre confit, acier et sucre "(Gestern), ces toutes premières compositions photographiques, qui ont signalé le point de départ pour plusieurs expositions ${ }^{2}$ et pour un catalogue intitulé Journal implicite (2013), sont donc " nées d'une nécessité : créer un monde imaginaire pour reprendre pied dans la réalité, transformer la douleur en beauté, l'aléa en élan » (Flem, Journal implicite, sans pagination). Dans l'enfance déjà, nous explique l'auteure, elle avait "besoin pour vivre de narrations, de fictions ». Elle était capable « de faire un personnage de n'importe quel bout de ficelle et de lui donner une âme et une histoire ").

Cette relation singulière aux objets « ordinaires », pour la plupart de l'expérience commune (biscuits, ciseaux, boutons...), ou cette capacité à en « investi[r] certains de manière très intime », parfois en élaborant un récit à partir d'eux, parfois en s'adressant à eux (pour ici reprendre notre interviewée à ce sujet), se retrouve aussi, chez Lydia Flem, dans ses livres dont la majorité est parue dans la collection "La librairie du XXI ${ }^{\mathrm{e}}$ siècle » auX éditions du Seuil. De ce point de vue, ses photos d'objets librement associés se donnent à "lire " comme indissociables de ses textes littéraires où se brouille la limite entre les êtres humains et les « choses de la vie » (selon ses mots), entre la réalité et l'imagination ou le souvenir. Par exemple, dans les quatre cent soixantedix-neuf fragments qui composent Je me souviens de l'imperméable rouge que je portais l'été de mes vingt ans (2016), " évident hommage à Georges Perec et délicieux exercice de remémoration centré sur les atours ", nous pouvons observer, avec Raphaëlle Leyris, que « Lydia Flem prouve [...] l'importance de ceux-ci - les pans de mémoire qu'ils charrient, ce qu'ils racontent de nous ». Pensons aussi à La Reine Alice (2011), " conte écrit à la troisième personne " (Dusaillant-Fernandes 252) et hommage discret à Lewis Carroll avec lequel Flem « me[t] en scène sa longue traversée intime et personnelle de la maladie » (Dusaillant-Fernandes 252). Dans cette œuvre est évoquée la décision prise par Alice de « se jouer » des cartes de jeu qu'elle a trouvées dans l'herbe sous un arbre. Cette "trouvaille » a été l'un des vrais déclencheurs du roman : comme l'auteure l'affirme ici, les choses matérielles, surtout celles que l'on trouve inopinément, peuvent bien ouvrir de nouvelles pistes de réflexion, donner envie d'« en faire quelque chose ». Un hasard semblable est à l'origine respectivement de deux livres dans lesquels notre auteure s'intéresse à sa propre famille, et où il lui arrive s'adresser à certains parmi les rares objets qui lui restent de ses parents. Ainsi que l'auteure le suggère ellemême dans ses œuvres, c'est une découverte particulière, faite en vidant la maison familiale, qui a déclenché l'écriture de Comment j'ai vidé la maison de mes parents, tout comme c'est l'ouverture des enveloppes et lettres gardées par ses parents qui a donné lieu à Lettres d'amour en héritage.

Lors de notre rencontre en mai 2019, il nous semblait important de revenir avec Lydia Flem sur son rapport aux objets ainsi que sur le rôle qu'ils jouent dans son travail, dans le processus créateur. Dans ce contexte, nous voulions mieux comprendre la manière dont l'auteure comprend, d'une part, son recours occasionnel à l'adresse écrite, en particulier dans Comment j'ai vidé la maison de mes parents et dans sa "lettre imaginaire " (ainsi qu'elle la désigne) à Paul Celan (Flem, "Lettre à Paul Celan »), et d'autre part, son exploitation textuelle de lettres-objets et de papiers privés, notamment dans L'Homme Freud et dans les premiers deux volumes de sa trilogie. Les belles réponses offertes à nos questions à ce sujet nous ont amenée à comprendre que, pour notre interviewée, comme elle le soutient elle-même ici, l'imagination est sa « seule maison ». 
KS : Dans votre Journal implicite (2013) sont publiées certaines de vos compositions photographiques faites à partir d'objets «trouvés au hasard d'une poche, d'un tiroir, sur le rebord d'une fenêtre, dans le désordre des jours et des lieux ». Les objets et la vie, avec ses désordres, sont-ils irrémédiablement liés, selon vous ?

LF : Les objets font partie de notre vie. Je pense que nous en investissons certains de manière très intime, qu'ils condensent nos émotions et nos pensées, notre mémoire. Mais je suis peut-être restée une enfant capable de faire un personnage de n'importe quel bout de ficelle et de lui donner une âme et une histoire. Aujourd'hui encore, il m'arrive de vouer un attachement singulier à un plume, à une lettre reçue, à un petit quelque chose gardé au fond de la poche. La limite entre les êtres humains et les choses me semble assez poreuse. Cette nuit, j'ai griffonné « L'imagination est ma seule maison » parce que je suis convaincue que les êtres humains ont besoin pour vivre de fictions, de narrations.

Quant aux désordres de la vie, c'est durant les mois où je recevais des traitements qui induisent des difficultés d'attention et de concentration - j'étais alitée, incapable de lire ou d'écrire plus de quelques phrases -, que je suis devenue photographe. Mais je n'ai pas cherché à saisir le réel. J'avais, au contraire, besoin de prendre une distance. Depuis mon lit, j'ai assemblé des objets, un peu comme des associations libres - sur le divan ou dans les rêves -, pour essayer de raconter ce que les mots ne pouvaient plus exprimer.

Le désordre des objets est une grande source d'inspiration. Un jour, en marchant dans un grand parc, j'ai découvert au pied d'un chêne un amas confus de cartes à jouer. Parce que j'avais beaucoup lu Lewis Carroll au moment d'écrire Comment je me suis séparée de ma fille et de mon quasi-fils, la découverte de ce jeu de cartes disséminées autour de cet arbre a été un des déclencheurs du roman, La Reine Alice. Dans Alice au pays des merveilles, Alice prend conscience que tout ce qu'elle a cru vivre avec les personnages d'un jeu de cartes était les feuilles qui tombaient de l'arbre en-dessous duquel elle s'était endormie auprès de sa sœur. Elle avait mélangé le rêve et la réalité. Pour ma part, quand j'ai aperçu les cartes sur l'herbe, j'ai eu le sentiment qu'elles surgissaient du livre de Lewis Carroll, qu'elles venaient me faire signe pour dire le mélange intime entre littérature et réalité. Qu'il fallait que j'accueille ce désordre, mêlé de hasard, que j'en fasse quelque chose à mon tour.

KS : Parmi les objets, dont le Journal implicite montre les photos, figurent des lettres autographes et enveloppes adressées, notamment : une enveloppe blanche adressée à quelqu'un habitant Saint-Germain-laBlanche-Herbe (photo "Au retour de la Blanche-Herbe »); et une enveloppe Kraft grand format (photo «Archive ») réunissant des lettres reçues, toujours dans leurs enveloppes...

LF : En janvier 2011, à la veille de la parution de La Reine Alice, j'étais encore assez vacillante mais très motivée d'accepter l'invitation de l'IMEC (Institut Mémoire de l'édition contemporaine) de faire, en quinze séances, une lecture intégrale de mon livre, filmée par Alain Fleischer. Olivier Corpet me proposait également d'exposer, dans un accrochage d'Alain Fleischer, douze autoportraits et douze compositions photographiques d'objets, qui avaient donné naissance au roman. C'est là, à l'IMEC, aujourd'hui dirigé par Nathalie Léger, à Saint-Germain-la-Blanche-Herbe, dans la campagne près de Caen, que j'ai reçu la lettre, avec cette adresse pleine de poésie sur l'enveloppe, dont j'ai publié une photographie dans le Journal implicite. Pendant quelques années, l'expéditeur de cette enveloppe, un lecteur, m'envoyait des lettres - sachant que j'intervenais quelque part - il m'écrivait au lieu où j'allais me retrouver...

Pour ce cycle de lectures, une main espiègle avait glissé un petit objet dans chacune des grandes enveloppes de couleurs vives, achetées à Rome. À chacune des quinze séances de lecture (à voir sur YouTube), j'ouvrais une enveloppe, je sortais les feuilles du tapuscrit, je découvrais l'objet-surprise (coquillage, boîte à musique, crayons, ...) puis, je lisais. Ensuite, pendant plusieurs mois, j'ai composé une série de quinze photographies, correspondant aux quinze étapes de la lecture-performance de La Reine Alice, à partir de divers objets et du manuscrit, devenu une sorte d'origami, découpé en échiquier, tissé, déchiré en mots et lettres, en jouant du va et vient entre littérature et plasticité, images et imagination, le roman revenant à ce qui l'avait fait naître, à sa source : la photographie.

La photographie extraite de la série «Pitchipoï et cousu main » dans le Journal implicite où on voit une main qui tient une enveloppe dans laquelle il y a des lettres, j'avoue que je ne les ai pas encore ouvertes. C'est une chose qui me reste à faire un jour. Ce sont de vieilles lettres, adressées par ma grand-mère à mon père lorsqu'il était enfant et adolescent. Mon père a perdu sa mère pendant la guerre. Elle a été déportée des PaysBas. À l'époque où ils s'écrivaient, mon père habitait chez un oncle à Bruxelles, n'ayant pas pu s'installer en Hollande avec sa mère et son frère en raison des lois de l'immigration. 
Parce que les lettres sont écrites en allemand, en caractères gothiques, leur lecture va me demander beaucoup de temps. Je pourrais faire appel à des spécialistes pour déchiffrer l'écriture. Mais je trouverais difficile d'inviter des inconnus dans l'intimité de cette correspondance. Car ce sont des objets de papier que j'imagine porteurs non seulement d'une histoire d'archive familiale, mais aussi de beaucoup d'émotion. De son vivant mon père n'a jamais mentionné ces lettres, dont la lecture sera sûrement bouleversante. Je les ai découvertes au moment de vider la maison de mes parents. J'ai aussi trouvé des journaux intimes de ma mère que je n'ai pas lus...

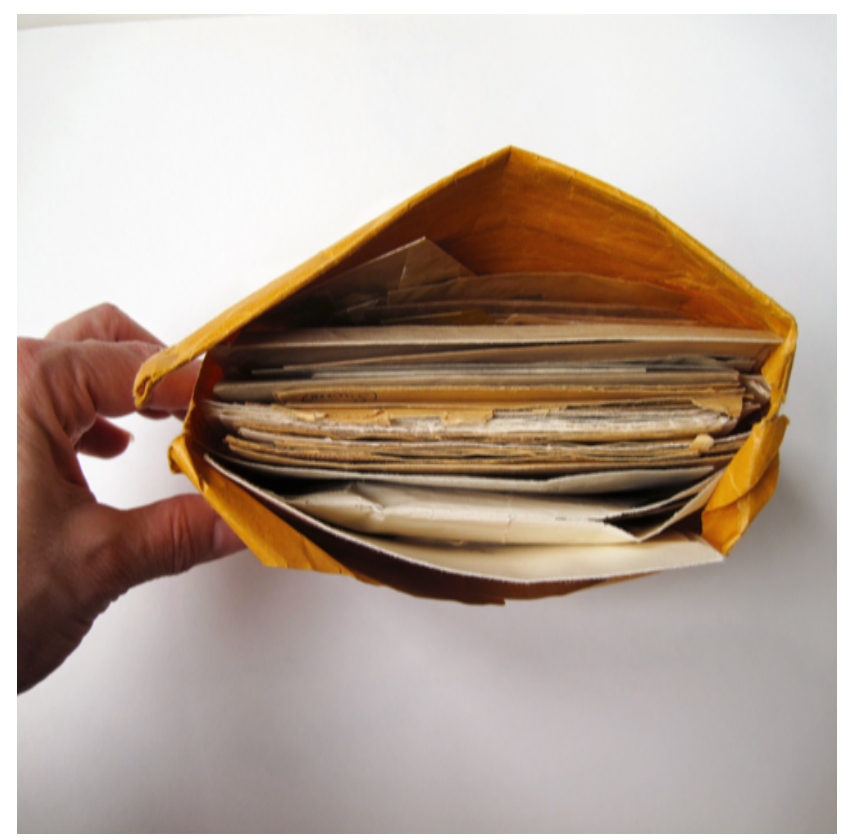

Flem, Lydia. « Archive », de la série « Pitchipoï et Cousu-main ». 2012, Galerie Françoise Paviot, Paris.

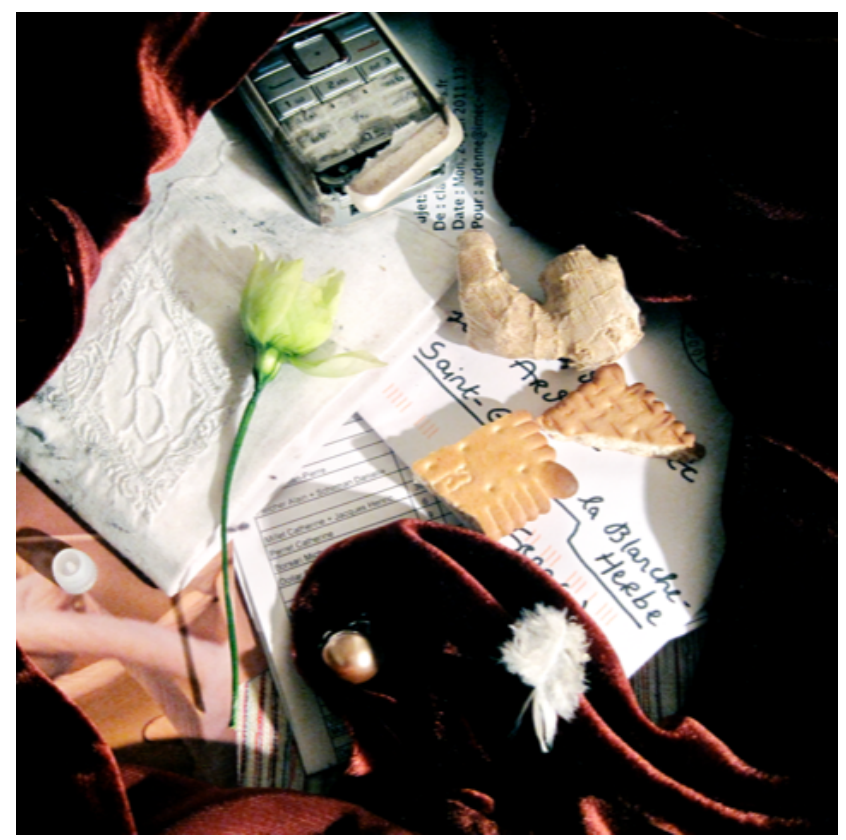

Flem, Lydia. « Au retour de la Blanche-Herbe », de la série « La Reine Alice ». 2011, Galerie Françoise Paviot, Paris. 
KS : Et les lettres d'amour de vos parents, auxquelles est consacré Lettres d'amour en héritage?

LF : Ce que je trouve très émouvant dans ces lettres, c'est qu'elles sont comme de petites valises. Matériellement, ces lettres sont toujours dans mon bureau, dans deux boites. Mon compagnon me dit qu'elles seraient peut-être plus en sécurité à l'IMEC, dans mon fonds d'archives, si je pouvais me séparer d'elles, mais je n'arrive pas encore vraiment à m'en éloigner physiquement. J'aime bien les sentir à proximité.

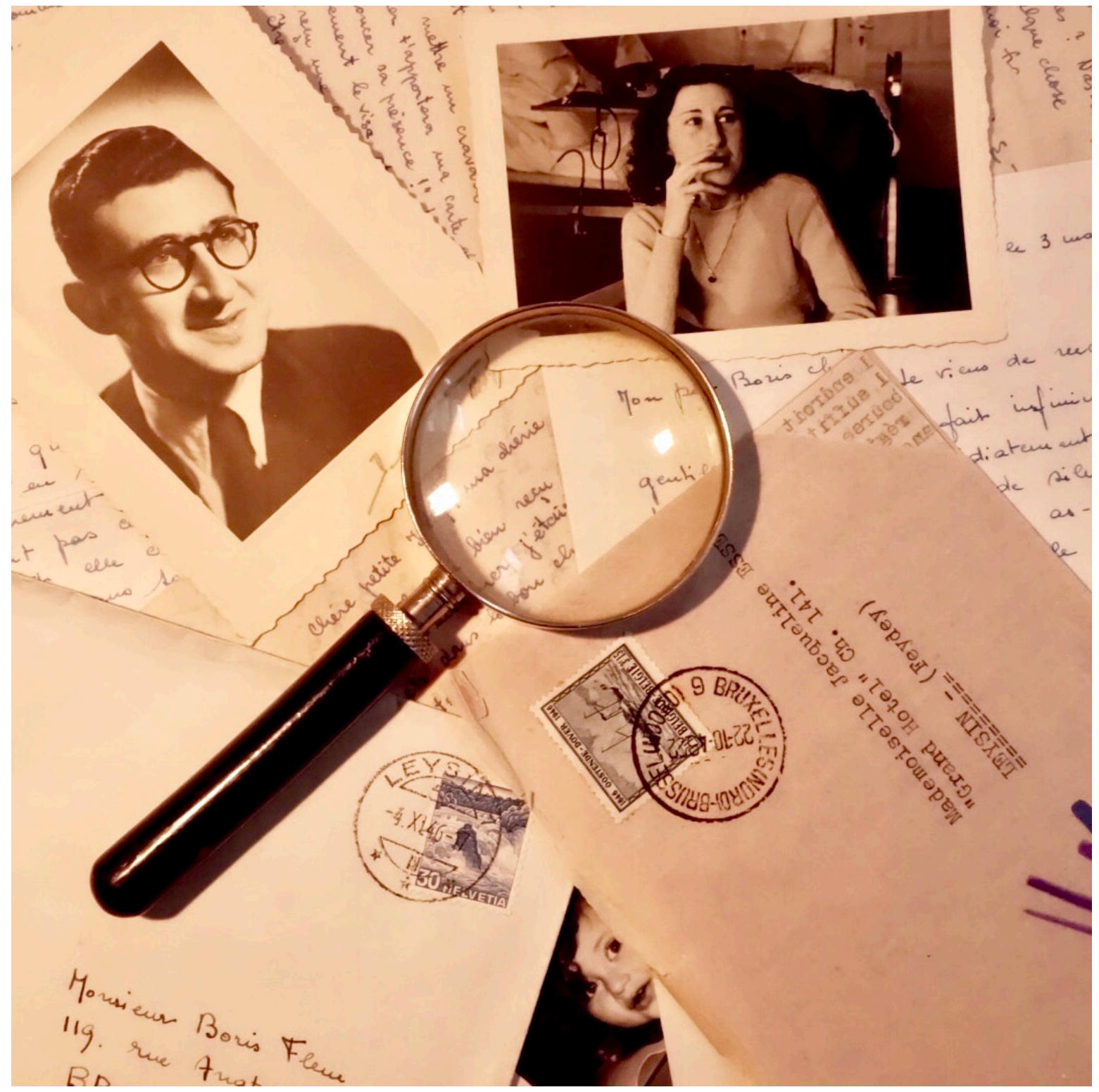

Flem, Lydia. «Sous la loupe (Lettres d'amour en héritage) ». 2015, photographie inédite, reproduite avec l'autorisation de l'artiste.

KS : Parmi tous les objets-souvenirs qui vous restent de vos parents, leurs lettres d'amour, découvertes au moment de vider le grenier de leur maison, occupent pour vous une place unique. Elles vous ont permis " d'entrer à nouveau en relation avec eux, de les toucher, en quelque sorte, en effleurant les enveloppes et les feuilles de papier sur lesquelles ils avaient écrit » (Lettres d'amour en héritage 21). Pourriez-vous commenter plus longuement votre rapport à ces lettres? 
LF : À l'instar des robes que ma mère a confectionnées de ses mains, sur lesquelles il m'arrive de chercher les points de couture, - dont les fermetures-éclairs se confondent, dans mon regard, avec les rails de chemin de fer de la déportation - les lettres portent la mémoire des gestes de la main. Françoise Héritier parlait des humeurs du corps. La graphie, la manière dont on tient un crayon, un stylo, et dont on l'appuie sur la feuille de papier, la danse des lignes, tout cela témoigne d'une inscription très personnelle du corps. Or, ce que je constate en lisant leurs lettres de jeunesse, c'est que mes parents ont gardé la même écriture toute leur vie, que la façon dont ils écrivaient avant ma naissance correspond à la façon dont ils écrivaient pendant le temps que j'ai partagé avec eux. D'où mon impression de retrouver quelque chose de la présence corporelle de mes parents...

KS : Dans Comment j'ai vidé la maison de mes parents, il vous arrive de vous adresser à ces objets reçus en héritage que sont les serviettes de papiers gaufrés, imprimés, à motifs de Vichy rouge et blanc, marqués de noms exotiques, de lieux lointains, de slogans, de dessins ; papiers qui seraient donc des petits billets, des missives ou des pense-bêtes. Vous écrivez : « [J]e vous ai ramenés sur ma table de travail. À la manière d'un classement de Perec ou d'un inventaire de Prévert, vous vous enchaînez les uns aux autres. Je ne pouvais vous précipiter dans le vide sans prendre note de l'étrange chapelet que vous formiez » (130). Qu'y a-t-il dans ces papiers qui ait pu vous inciter à les vouvoyer?

LF : C'est un moment clé que vous soulignez, moment qui correspond au vrai début du livre. Depuis quelques jours, je tentais de vider la maison de mes parents. Je tombe alors sur une boite qui contient des serviettes en papier que ma mère avait ramenées de différents endroits, d'une crêperie, d'un restaurant, d'un café, d'un coin ou l'autre de France ou d'Europe. Ces papiers, sur lesquels elle avait écrit ses pensées, ou parfois tout simplement un lieu et une date, sont une trace de sa vie, de ses voyages, de sa graphie. En les lisant, j'avais l'impression que ma mère me parlait. Me parlait d'elle, de mon père, et éventuellement, des personnes qu'ils rencontraient en voyageant.

La serviette en papier avec le logo et le nom du restaurant, avec ce qu'on peut écrire dessus, c'est proche d'un objet de correspondance. La découverte de ces serviettes a été le déclencheur de l'écriture de Comment j'ai vidé la maison de mes parents. En les lisant, j'avais le sentiment d'assister à une conversation. Comme si ces bouts de papier venaient me chuchoter à l'oreille les histoires de mes parents, de leurs voyages à deux, de leurs rencontres. Comme si, réunis ensemble, ces objets étaient les témoins presque vivants de leur existence en dehors de la mienne...

KS : Et le vouvoiement ? Peut-on lire dans votre adresse la preuve d'un attachement singulier ?

LF : Leurs objets devenaient les ambassadeurs, les porte-paroles ou les véhicules, de mes parents disparus. A travers eux, je m'adressais à mon père et à ma mère, dans une sorte de dialogue au-delà de leur mort. Ce dialogue imaginaire ne cesse jamais, je pense. C'est pourquoi les archives sont tellement précieuses...

KS : Théoriciens et critiques de la correspondance affirment qu'écrire une lettre, c'est donner de soi. Avec leurs lettres, et sans le savoir, vos parents vous auraient-ils donné quelque chose ? En écrivant Lettres d'amour en héritage, cherchiez-vous alors à faire contre-don, à remercier ou, à tout le moins, à " rendre » hommage, si nous admettons la part de don inhérente à l'expression?

LF : Avec Comment j'ai vidé la maison de mes parents, qui est le premier livre que mes parents n'ont pas connu, mais aussi les Lettres d'amour en héritage, je cherchais à leur rendre hommage et, sans doute aussi, à poursuivre la conversation avec eux. Le dialogue qui suit la disparition n'est pas le même que celui qu'on a eu de leur vivant, il devient plus apaisé et plus heureux. II est peut-être idéalisé... Leurs lettres de rencontre représentaient une transmission de valeurs, la puissance de l'amour qui dure, Eros contre Thanatos. Un très précieux don.

KS : Vous disiez avoir relu Lewis Carroll (peut-être dialogué avec lui ?) pour Comment je me suis séparée de ma fille et de mon quasi-fils. Et dans l'affirmation suivante, extraite de La Reine Alice, nous trouvons un hommage, peut-être une reconnaissance de dette, à l'auteur d'Alice au pays des merveilles : " Je suis née de l'imagination de Lewis Carroll, mais dans une lettre posthume à Alice Liddell, devenue Mme Hargreaves [...], il m'encourageait à devenir écrivain à mon tour ». D'une manière ou d'une autre, tous vos livres sont-ils par essence des hommages, discrètement adressés? 
LF : Toute petite, et peut-être même avant d'apprendre à lire, quand on me racontait des histoires, je rêvais de devenir écrivain, d'appartenir à ce monde-là. J'avais une admiration folle pour les écrivaines et les écrivains. Enfant unique, je ne me sentais jamais seule, parce que les livres étaient mes amis. Lire une histoire, c'est un peu comme découvrir de vieilles correspondances : c'est dialoguer avec des absents qui sont tout de même encore présents, par leurs mots et leur écriture. Aujourd'hui encore, j'ai le sentiment que les auteurs me font un immense cadeau avec leurs écrits - lettres ou livres. Je leur suis infiniment reconnaissante, sans la littérature et les artistes, je ne pourrais simplement pas vivre. Je leur dois tout.

KS : Vous-même vous avez adressé à Paul Celan, l'un des plus grands poètes de notre temps, ce que vous désignez comme une "lettre imaginaire " (Lettres d'amour en héritage 86). Dans cette lettre, publiée dans le numéro 33 (2015) des Moments littéraires consacré à votre œuvre, vous tutoyez le poète et vous l'appelez par son prénom, peut-être parce que votre mère l'a connu dans sa jeunesse. Vous écrivez : « Pardonne-moi, Paul, de t'écrire, mais je n'ai personne d'autre à qui m'adresser » (75). Que vous a permis cette lettre, et plus particulièrement, ce dispositif d'adresse?

LF : Paul Celan est un des plus grands poètes du $X X^{e}$ siècle, son œuvre tente de mettre des mots sur l'ineffable du génocide des Juifs sous le régime nazi. Chercher un dialogue imaginaire avec lui, c'était tenter d'élaborer quelque chose du traumatisme reçu en héritage alors même qu'il n'était pas aisé d'avoir cet échange avec ma mère, revenue d'Auschwitz. Plusieurs circonstances m'y encourageaient : ma mère avait connu Paul Celan à Tours, avant la guerre. Après la guerre, il avait cherché à prendre de ses nouvelles via une lettre à un ami commun, mais ma mère ne l'a su que vingt ans après son suicide en 1970. Ils avaient été très proches - une photographie en témoigne, qui a été publiée dans la Correspondance de Paul Celan avec son épouse, Gisèle Lestrange ; c'est, en effet, une des photos connues de Paul Celan en France avant la guerre.

L'introspection et la psychanalyse conduisent presque toujours, à un moment ou à un autre, à vouloir se déprendre de ses fantasmes, à les confronter avec sa généalogie, pour retrouver son propre souffle. M'adresser à Paul Celan, c'était essayer de tisser des mots, trouver du sens, au-delà du silence maternel. Esquisser, par ce détour, une forme de réparation.

KS : À l'égard de vos parents, vous dites, dans Lettres d'amour : « Ils ne sont plus là pour connaître mes joies et mes peines. En pensée, je les leur raconte. Déménagement, mariage, livre, anniversaires, voyages, les enfants qui grandissent, toutes les choses de la vie » (253). Dans la mesure où vous « racontez » dans Lettres d'amour vos impressions de lecture, vos constats en lisant leurs lettres d'amour, ce livre est-il quelque part, pour vous, une lettre écrite à vos parents?

LF : Oui, je crois que je m'adresse à mes parents, en faisant alterner dans mon livre des fragments de leurs lettres d'amour et mes commentaires concernant ces lettres. Et il est possible qu'avec Lettres d'amour en héritage, je prolonge le dialogue entamé dans Comment j'ai vidé la maison de mes parents. Parce que, comme un lecteur me l'a fait observer lors d'une rencontre organisée dans une librairie, je n'avais pas mis de point final à Comment j'ai vidé la maison. Comme si, disait-il, je n'avais pas fini d'écrire sur les objetssouvenirs de mes parents. (Ce manque de ponctuation à la fin du livre était bien voulu de ma part. Pour toutes les traductions, j'ai dû me battre pour vérifier qu'ils n'avaient pas rajouté un point.) Un jour, en effet, je me suis rendu compte que j'allais donner une suite à Comment j'ai vidé la maison ; que, pour reprendre un tête-à-tête avec mes parents, j'allais revenir sur leurs lettres. De ce point de vue, les deux livres sont une manière de m'adresser à eux, pour essayer d'avoir un échange au-delà de la vie et de la mort, oui. Mes lettres posthumes, en somme.

KS : Vous dites que vous avez voulu « poursuivre le dialogue », en écrivant sur, et parfois avec, les lettres de vos parents. Pour vous, l'écriture est alors une sorte de substitut de la parole?

LF : Oui, absolument. L'écriture, disait Freud, est le substitut du corps maternel absent. L'écriture, c'est d'abord de la voix. J'écris d'ailleurs souvent à voix haute, les deux se mêlent intimement pour moi. Les photographies - comme celles que j'ai prises des robes que ma mère a cousues - permettent, elles aussi, de renouer avec nos absents. Elles sont elles aussi un moyen d'interroger l'histoire, d'enquêter sur le passé. J'ai récemment découvert, en regardant la légende d'une vieille photo, des circonstances autour de ma naissance que j'ignorais largement et dont je parlerai peut-être dans un prochain livre. Comme la vie, les archives nous réservent des surprises. 
KS : "Les habits collent à la peau », nous rappelle Je me souviens de l'imperméable rouge que je portais l'été de mes vingt ans. "Ils nous protègent et nous exposent [...] trahit notre part d'ombre " (quatrième de couverte). De ce point de vue, les habits se rapprochent-ils de la lettre, pensez-vous ? Avez-vous l'impression que, de manière générale, la lettre expose tout autant qu'elle dissimule ?

LF : Les vêtements sont, bien sûr, des signes que nous adressons, volontairement ou involontairement, aux autres. La lettre m'apparaît comme un espace de méditation et d'introspection, qui, consciemment ou à notre insu (lapsus, ratures, associations involontaires...) nous révèle, nous raconte, davantage, je crois, qu'elle ne nous dissimule. Bien sûr, comme dans tout échange humain, l'interprétation demeure ouverte et multiple.

KS : Dans les archives, selon l'historienne Arlette Farge, «il est tant de formes calligraphiées qui étonnent » (10) pour le chercheur travaillant aujourd'hui à partir de petits mots archivés dans des boîtes. Pour sa part, elle trouve particulièrement saisissants les messages tracés de façon quasi phonétique » et la signature de ceux qui ne savent pas écrire. À part la surprise de découvrir, en lisant les lettres de vos parents, " que les traits de leurs caractères étaient fort semblable à ce que [vous aviez] connu d'eux " (Lettres d'amour 41), quel autre étonnement avez-vous ressenti ?

LF : Ce qui m'a touchée d'abord, c'est l'intensité d'une rencontre amoureuse, qui a lieu dans l'espace même de la correspondance, elle s'écrit en se vivant et se vit en s'écrivant. J'ai compris en les lisant que je suis née de leurs échanges de lettres. Ensuite, j'ai été émue de découvrir qu'ils avaient choisi la langue française pour partager leurs sentiments ; ils auraient très bien pu s'écrire en allemand, mais sans doute, c'était comme pour Paul Celan, une langue de la mort.

Un autre étonnement, c'est le nombre de lettres qu'ils échangeaient, parfois plusieurs missives dans la même journée. Leur correspondance était au centre de leur vie. Ils s'envoyaient aussi des coupures de presse, des échantillons de tissu, des petits ajouts divers qu'ils glissaient entre les feuilles de papier à lettres comme de petits cadeaux qu'ils s'offraient, une manière de ne jamais se quitter. Ils se donnaient d'ailleurs des rendezvous pour écouter des émissions radiophoniques - notamment des concerts - à la même heure. C'était aussi très touchant de voir qu'ils avaient numéroté chaque lettre, toutes précieusement conservées, presque intactes, comme si elles venaient d'être apportées par le facteur, encore à décacheter.

KS : Depuis 1986, des correspondances inédites de Freud ont été publiées et traduites en français, notamment celle avec Karl Abraham (Correspondance complète, 1907-1926, trad. Fernand Cambon). Après La Vie quotidienne de Freud et de ses patients ([1986] 2018), pour lequel vous avez consulté certaines de ces correspondances, puis avec L'Homme Freud (1991), vous vouliez "suivre Freud à la trace, l'accompagner dans ses voyages au pays de nulle part, lire pardessus son épaule " (préface). En quoi la lecture des correspondances de Freud vous-a-t-elle aidée dans ce sens, pour ce projet de livre ? Écrire en citant des lettres, est-ce pour vous faire un bout de chemin avec les écrits d'autrui ?

LF : Absolument. Nous étions avant Internet. Je revois mon bureau à l'époque, Le sol était couvert des correspondances de Freud. Parce qu'il écrivait régulièrement une dizaine de lettres le même jour, à divers correspondants, j'aurais aimé pouvoir les comparer, voir l'influence du destinataire sur sa pensée.

Avec L'Homme Freud, je voulais raconter l'inventeur de la psychanalyse autrement que par ses ouvrages théoriques. Faire appel à ses lettres, ce n'était pas, me semblait-il, le regarder par le petit bout de la lorgnette mais, au contraire, lui rendre hommage dans l'exacte mesure où il était un théoricien du quotidien. C'est justement parce qu'il reconnaissait aux choses les plus minuscules, ordinaires, quelconques de notre vie, leur importance, que je me suis autorisée à écrire les livres que j'ai écrits, avec lui d'abord, puis sans lui ; de rendre toute sa noblesse à la vie quotidienne, dont la lettre fait partie. Freud en a écrit environ 20.000. J'ai eu très rapidement le sentiment que c'était le matériau idéal pour entrer dans son « atelier », son work in progress.

La vie s'écrit dans les lettres, dans les billets, dans tout ce qu'on peut laisser comme trace écrite ou imprimée. Aujourd'hui, la vie laisse ses empreintes dans les mails et les textos. En raison de la valeur que peut représenter une correspondance, même électronique, je suis heureuse d'avoir imprimé un grand nombre de mails, et je regrette ceux que je n'ai pas conservés. Depuis des années, j'ai une correspondance (à la fois postale et numérique) avec un ami qui a eu un parcours très différent du mien, chirurgien et grand voyageur. En relisant nos échanges j'ai l'impression qu'elle forme une double introspection, chacun s'y raconte comme si l'amitié devenait le support d'une psychanalyse à deux faces ou une sorte de journal intime, croisé et parallèle. 
KS : Écrivaine et photographe, vous arrive-t-il de recevoir du courrier de vos lecteurs ?

LF : Comment j'ai vidé la maison de mes parents est un livre qui, à ma très grande surprise, continue à faire son chemin. Sa réception m'étonne parce qu'en l'écrivant j'avais l'impression de dire des choses dont personne ne parlait, qu'on n'avait même pas le droit d'évoquer. Or dans leurs lettres, les lecteurs me racontent comment, eux, ont vidé la maison de leurs parents, ce qu'ils ont découvert, avec parfois des photos à l'appui, des textes, des extraits de journaux. J'aime l'idée que chacun peut s'emparer de mes livres, peut se les approprier, pour son propre compte, comme un miroir. La Reine Alice, également, a suscité énormément de confidences personnelles, parfois des confidences déchirantes. Je sens une obligation morale d'y répondre, comme les lecteurs m'écrivent aux éditions du Seuil, qu'il faut du temps pour qu'elles me parviennent, puis du temps pour y répondre, je m'en veux régulièrement du délai que je laisse passer. Je reçois ces lettres comme des cadeaux.

KS : À l'époque où vos parents s'écrivaient des lettres d'amour, votre mère, dites-vous, " attendait chaque distribution de courrier avec impatience " (Lettres d'amour 37). Sommes-nous aujourd'hui nostalgiques, pensez-vous, d'une époque où on pouvait attendre avec impatience l'arrivée du courrier?

LF : Attendre, ce n'est plus dans l'air du temps. Au contraire, il semble que l'impatience se généralise. Un plan cinématographique, jadis, durait autour de 12 secondes, aujourd'hui avant 2,5 secondes, le public se lasse. Peut-être va-t-on revenir un jour aux joies de la lenteur, par goût, ou par nécessité...

KS : Pour finir, accepteriez-vous de nous parler un peu de votre projet de livre actuel ?

LF : Je peux dire que je suis en train de faire une grande collecte d'archives dont j'espère pouvoir tracer une sorte d'autobiographie au pluriel.

\section{Notes}

${ }^{1}$ Nous remercions très sincèrement Fanny Leveau, doctorante en études françaises à l'Université Western Ontario, pour son aide précieuse dans la transcription de cet entretien.

${ }^{2}$ Lydia Flem a exposé ses photos à Caen, à l'Institut de la mémoire de l'édition contemporaine IMEC (en 2011), à l'espace photographique Contretype, à Bruxelles (en 2011-2012), à la Biennale de la Photographie à Berlin (en 2014), et à Paris, à la Maison européenne de la Photographie (en 2015).

\section{Bibliographie}

Bonnefoy, Yves, Alain Fleischer, Fabrice Gabriel, et al. Les photographies de Lydia Flem. The Photographs of Lydia Flem. Volume issu d'une rencontre à la Maison européenne de la photographie le 23 octobre 2013, d'une rencontre à la Maison de l'Amérique latine le 12 novembre 2013 et de l'exposition de Lydia Flem, Journal implicite, à l'Institut français de Berlin du 17 octobre au 22 novembre 2014. Maison européenne de la photographie/Maison de l'Amérique latine/Institut français Berlin, 2014.

Celan, Paul et Gisèle Celan-Lestrange. Correspondance (1951-1970). Seuil, « La Librairie du XXI ${ }^{\mathrm{e}}$ siècle », 2001.

Dusaillant-Fernandes, Valérie. « Le cancer au pays d'Alice : Lydia Flem et son conte à ne pas mourir debout. » Interférences littéraires/Literaire interferenties,18, mai 2016, pp. 251-267.

Farge, Arlette. Il me faut te dire. Éditions du Sonneur, coll. « Ce que la vie signifie pour moi », 2011. 
Schwerdtner, Karin. « Lydia Flem : 'L’imagination est ma seule maison’. Entretien autour des lettres (et) objets » Nouvelle Revue Synergies Canada, Nº 13 (2020)

Fleischer, Alain et Lydia Flem. «La Reine Alice. Lecture-performance dans la Grange-aux-Dimes en l'Abbaque d'Ardenne à l'Imec les 27, 28 et 29 janvier 2011. »YouTube, téléchargé par Art Solilok, le 4 janvier 2015, www.youtube.com/watch?v=nW_rRr1IPD0.

Flem, Lydia. Casanova ou l'exercice du bonheur. Seuil, « La librairie du XXI ${ }^{\mathrm{e}}$ siècle », 1995.

---. Comment j'ai vidé la maison de mes parents. Seuil, « La librairie du XXI ${ }^{\mathrm{e}}$ siècle », 2004.

---. Comment je me suis séparée de ma fille et de mon quasi-fils. Seuil, « La librairie du XXI siècle », 2009.

---. L'Homme Freud. Une biographie intellectuelle. Seuil, « La librairie du XXI ${ }^{\mathrm{e}}$ siècle », 1991.

---. Je me souviens de l'imperméable rouge que je portais l'été de mes vingt ans. Seuil, « La librairie du $\mathrm{XXI}^{\mathrm{e}}$ siècle », 2016.

---. Journal implicite. Photographies 2008-2013. Éditions La Martinière, 2013.

---. « Lettre à Paul Celan. » Les Moments littéraires, 33, 2015, pp. 73-80 (première mention dans Lettres à l'amant, dix-sept lettres d'écrivains au féminin. Colophon Imprimeur, Grignan, 1997).

---. Lettres d'amour en héritage. Seuil, « La librairie du XXI ${ }^{\mathrm{e}}$ siècle », 2006.

---. La Reine Alice. Seuil, « La librairie du XXI ${ }^{\mathrm{e}}$ siècle », 2011.

---. La vie quotidienne de Freud et de ses patients. Hachette, 1986 ; Seuil, 2018.

Gestern, Hélène. « Journal implicite (Lydia Flem). » La Faute à Rousseau, 69, juin 2015, p. 74.

Héritier, Françoise. Les deux sœurs et leur mère. Odile Jacob, 1994.

---. Masculin/féminin. La pensée de la différence. Odile Jacob, 1996.

Leyris, Raphaëlle. "Lydia Flem, de ce côté du miroir. » Le Monde des livres, le 11 avril 2016 , www.lemonde.fr/livres/article/2016/04/13/lydia-flem-de-ce-cote-du-miroir_4901355_3260.html 